\title{
AVID: An intelligent CAI system for small computers
}

\author{
K. W. SCHOLZ and L. H. SNOW \\ Medical College of Pennsylvania, Philadelphia, Pennsylvania 19129
}

\begin{abstract}
A computer-assisted instruction (CAI) system which includes facilities for interactive graphics and for limited natural language processing has been implemented on a DEC PDP-11 minicomputer. Lesson authoring aids permit inexperienced authors to create lessons with ease and permit transporting lessons to and from other systems. The system supports eight simultaneous independent users and provides facilities for student performance evaluation and record management.
\end{abstract}

AVID (an acronym for Academic and Vocational Interactive Dialog) is a general-purpose system for Computer-Assisted Instruction (CAI) implemented on the Digital Equipment Corporation (DEC) PDP-11 under the RT-11 operating system. Although AVID was developed in a medical environment, it has been designed for application to a wide range of academic or vocational education needs. The system design has been directed toward three primary goals: to provide maximum responsiveness to student needs, permit lesson development by inexperienced authors, and keep hardware costs to a minimum.

Responsiveness to student needs is provided by a natural language question-answering facility. The facility permits a student to enter a query as a simple English question in order to locate curricular material located anywhere in the system's data base. Lesson development is eased by providing an authoring option in which the dialogue is system driven (e.g., the entry of each component of the lesson is prompted). This facility permits a content-area expert with little or no computer experience to generate quality lessons with a minimum of training. CAI system costs are kept to a minimum by designing AVID to run on a small minicomputer rather than timesharing on a full-sized computer. AVID could service several terminals on a system as small as a DEC LSI-11 or a Heath $\mathrm{H}-11$ for a total hardware cost under $\$ 5,000$.

AVID will be described from several perspectives: the student's view of the system, the authoring aids and the authoring process, the data structures and algorithms which permit implementation of a com-

Send requests for reprints to Dr. K. W. Scholz, Medical College of Pennsylvania, 3300 Henry Avenue, Philadelphia, Pennsylvania 19129. The authors wish to acknowledge the contribution of Frank Smith and Beatrice Holly for their many valuable suggestions and criticisms during the design and implementation of this system. plex system on a small minicomputer, and the academic and pragmatic justification for AVID's development.

\section{AVID FROM THE STUDENT'S PERSPECTIVE}

The mechanics of student interaction with AVID have been kept as simple as possible. In addition to the traditional LOGON and LOGOFF functions found on any timesharing system, a student need learn only three simple directives to fully utilize the system. The commands each consist of two key strokes: first pressing the ESC key, and then typing a single key signifying the command. The command "ESC-F" forces AVID to exit immediately from the current lesson even if that lesson has not been completed. The command "ESC-B" directs AVID to back up to the previous display or "frame." The command "ESC-ESC" directs AVID to exit the current lesson and back up to a previous lesson from which a student exited by asking AVID a question. Any line of input to the system is terminated by pressing the RETURN or ENTER key (depending on the terminal), and typing errors may be immediately corrected using a DELETE key.

Other than these few mechanical operations, the remaining interaction between AVID and the student consists of a simple English dialogue. Immediately upon LOGON, the student is asked for the name of a lesson. At this point, or at any subsequent point in a lesson, the student may answer a question with another question, such as "Do you have any lessons on biochemistry?" or "What information do you have on electronic theory?" AVID will attempt to locate information anywhere in its data base which appears to be germane to the student's question. If only a single lesson or portion of a lesson appears relevant, that information is immediately displayed. A more general question that suggests a small number of specific topics (less than 20) causes a num- 
bered list of the topics to be displayed for the student's choice. If a question is too general (e.g., "Can you tell me about medicine?"), the student will be cautioned to be more specific.

Whenever a student question directs AVID into a lesson, the previous lesson or "frame" from which the question was asked is "remembered" in the event the student wishes to return to it. This memory facility is recursive to 10 levels. Once a student is satisfied that his question has been answered, the ESC-ESC command described above can be used to back up to each previous frame from which a question was asked.
The remaining student-system interaction is under the explicit control of the lesson author. Each frame in a lesson presents information as sentences, paragraphs, or graphics figures and solicits input from the student. His response may be a letter, a number, a phrase, or a short sentence. The lesson author will have provided a collection of response alternatives which AVID uses to direct the student to the frame appropriate to his response. This normal response-contingent dialogue is maintained at all times unless the student chooses to deviate from it using the query facility described above.

Figure 1 illustrates photographically the operation

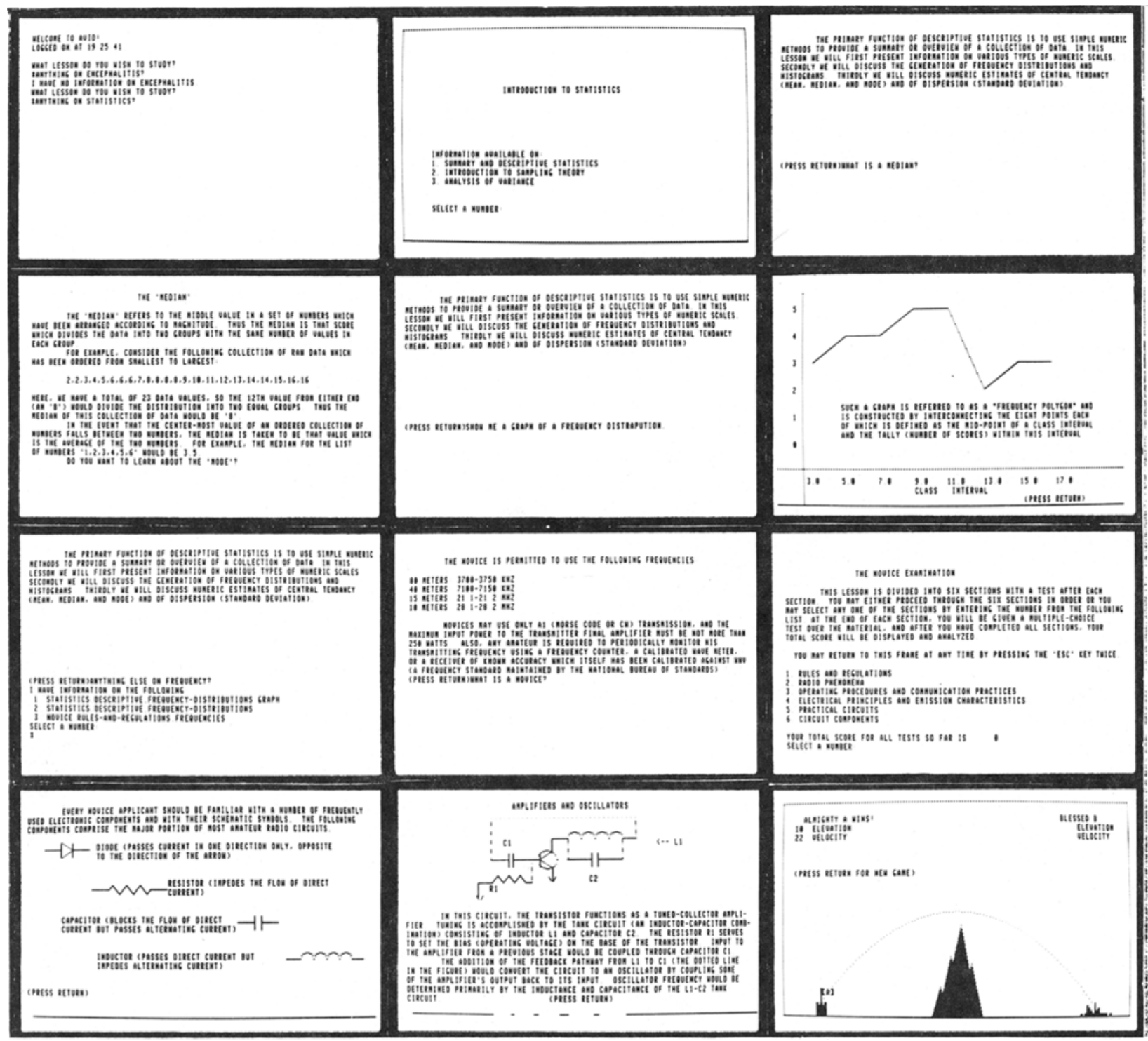

Figure 1. An example of student-AVID interaction. (Read panels left to right, top to bottom.) The student begins after logging on by seeking information on his latest lecture. Finding nothing, he inquires about statistics and is transferred to the start of a statistics lesson. After finding his way to information about the median (Panel 4), he backs up (ESC-B) and requests information on frequency distributions. Despite his spelling error, he is shown a frequency polygon. The student then exits from this display (ESC-ESC), and in response to his next less specific question finds information on frequency in a totally unrelated lesson (Panels 7 and 8). Curious, the student browses in the lesson on radio theory until discovering a circuit diagram. Finally overwhelmed, he decides to relax and try his hand at a game of "Potshot." 
of the system from the viewpoint of a curious (but somewhat distractable) student.

\section{AVID LESSON PREPARATION FACILITY}

The AVID Lesson Preparation Facility (LPF) provides two distinct methods of lesson preparation. First, the LPF may be executed interactively by maintaining a dialogue with the author much as a completed lesson communicates with a student. In the second method, the AVID text editor (a facility totally independent of the LPF) may be utilized to prepare a lesson source file for subsequent compilation by the LPF. The source file may consist of an entire lesson, portions of several lessons, or corrections and additions to an existing lesson.

\section{Lesson Structure}

The conceptual view of a lesson is the same for either programming method. Each lesson consists of an indefinite number of frames, where a frame is the unit of information displayed at one time to a student. One frame may comprise as little information as a screen erase or a single character, or as much as a "screenful" of text $(1,920$ characters $)$ or graphics illustrations. From an author's viewpoint, each frame must first be given a name. The frame's name or "frame title" constitutes an important component in AVID's data structures. Each frame title consists of a number of keywords that label the contents of the frame. The keywords are ordered from most general to most specific. The most general, or leftmost, keyword identifies the lesson topic, and subsequent keywords refine the title down to the specific topic of the associated frame. In the event a frame represents a continuation of the theme of a preceding frame, integers (rather than words) are suffixed to the rightmost keyword. In essence, each keyword in a frame title identifies a level in an arbitrary semantic hierarchy and the collected set of frame titles organizes the entire curricular data base as a semantic network.

\section{Frame Structure}

Each frame consists of three components, any two of which may be omitted. Part 1 , the text block, consists of the text or graphic information that is to be displayed to the student. Part 2, the calc block, consists of an indefinite number of lines of calculations, function calls, string manipulations, or conditional branches (within the calc block) which are entered in a syntax similar to BASIC or FORTRAN. Arithmetic expressions may be entered using fully parenthesized infix notation, and the available functions include facilities for student record manipulation, for interactive graphics, and for generating random numbers and timed delays. Part 3, the response block, contains a number of anticipated responses, each associated with the title of the frame to be displayed if that response is selected by the student. Various pattern-matching options are provided to allow the anticipated response to match student spelling errors or synonyms.

\section{Authoring Process}

If an author elects to use the interactive capability of the LPF, he will be explicitly prompted for each component of the frame. If he responds to the prompt for a frame title with the name of an existing frame, he is given the options of ignoring, copying, modifying, deleting, replacing, or inspecting that frame. If he elects to replace or create a frame, each of the three sections is prompted. The author may respond to any prompt with a null line, in which case that section is terminated and the next section prompted. Any frame titles introduced in the response block are either linked appropriately (if the frame already existed) or are added to a list of incomplete frames (if the frame does not exist). As each frame is completed, the list of incomplete frames is displayed as an aid to the author.

The distinction between operations performed in the calc block and in the response block deserve additional comment (see Table 1). The collection of statements in a calc block are very similar in form to existing algebraic programming languages, and, as such, provide an experienced programmer with a familiar powerful programming tool. The facilities for input and output from both the student's terminal and the disk plus the capability for conditional branching permit creation of a complete mini lesson within a single calc block if desired. However, the effective use of this facility requires that an author achieve a degree of programming competence that may well be beyond his desire or capability to acquire. The structure of any frame in AVID permits totally ignoring the calc block, while still providing a versatile response-contingent linkage to a number of other frames. Thus, an inexperienced author can still generate sophisticated highly interactive lessons by specifying only the text and response portions of each frame. With this facility, AVID extends the capability for lesson authoring to a far wider audience.

\section{AVID SYSTEM DESIGN SUMMARY}

AVID has been designed to run under Digital Equipment Corporation's RT-11 operating system on a PDP-11 computer with at least $16 \mathrm{~K}$ words of memory and one disk. RT-11 is a single-user twopartition real-time system. The AVID resident monitor executes in the foreground partition and provides fully timeshared interactive lesson execution for up to 16 students ( 8 with a $28 \mathrm{~K}$ word computer). 
Table 1

Sample Source Listing for Two Frames

TITLE: STATISTICS.DESCRIPTIVE.TEST.18

TEXT:

IF A DISTRIBUTION OF SCORES CONTAINED SEVERAL EXTREME SCORES, WHAT MEASURE OF CENTRAL TENDENCY WOULD BE MOST APPROPRIATE?

RESP:

MED $=$

YES

*MEAN $=$

.NOTMEAN

*MODE

NOTMODE

TITLE: STATISTICS.DESCRIPTIVE.TEST.18.YES

TEXT:

$$
\text { YES, THAT WAS CORRECT! }
$$

CALC:

$\mathrm{N}=\mathrm{N}+1$

$\mathrm{C}=\mathrm{C}+1$

WRITE "DO YOU WANT TO REVIEW?"

ACCEPT $S$

IF S-"YES", L,M

M: SAVE N,1

SAVE C,2

$\mathrm{N}=0$

L: $\quad \mathrm{C}=0$

RESP:

$>\mathrm{N}$

STATISTICS

$=$

Note-The first frame contains a title, the text block, and a response block (note that the calc block has been omitted). The response "MEDIAN" (or any response beginning with "MED") will result in a transfer to the frame STATISTICS. DESCRIPTIVE.TEST.18.YES. A frame title which begins with a period is understood to be suffixed to the title of the current frame. The second response matches any student response in which the letters "MEAN" appear. The third response matches any student response which ends with the letters "MODE." The second frame includes a calc block which, in this example, is used to conditionally save performance information in the student's record. The variable $N$ is used to count questions asked of the student, and the variable $C$ is used to tally correct answers. If the student indicates he does not wish to review, both variables are saved. Otherwise, the two variables are not saved, and the frame entitled "STATISTICS" is entered. Note that responses to this frame are processed in the calc block, not in the response block (the expression $>N$ succeeds only if the current value of the variable $N$ is greater than zero).

The RT-11 background partition is used exclusively for the AVID LPF. The foreground-background distinction is totally transparent to the user. A system with less than $16 \mathrm{~K}$ could provide either multiple student access or the LPF, but not both simultaneously. Memory allocation for a $28 \mathrm{~K}$ word (56K-byte) system is summarized in Figure 2.

\section{Structure of the AVID Data Base}

The data base, which contains all lessons in an arbitrarily large curriculum, is developed in two files on the random-access mass storage device. The first, or master, file consists of a header and a number of variable-size records (one record for each frame). The second file serves as the frame title library and is sequentially allocated with fixed-size records. The frame title library serves as a key or index to the master file and provides indexed sequential access to the master file for all search operations.

The details of the data structures are summarized in Figure 3. Each record in the master file contains a record header and a (possibly empty) record body. The header contains a link to its frame title, codes for specifying access and student record management, and the word count of the record body. The body contains (optionally) the frame text, the calc block (compiled into an intermediate code), and the response block. Each response in the response block includes links both to the frame title library and to the appropriate record in the master file. Disk space for new frames is allocated using an available storage pointer which is maintained in the master file header. Deleted or unwanted nodes are reclaimed using a conventional "garbage collection" algorithm.

\section{AVID System Monitor}

The AVID monitor timeshares among all currently active ports by context switching among ports at each I/O wait. A port table approximately $1.1 \mathrm{~K}$ words long is associated with each port which contains essential addresses, flags, and $\mathrm{I} / \mathrm{O}$ buffers. A context switch is effected by pointing a general register to the appropriate port table then flowing into code which is shared by all ports. [Details of the context switching algorithm are described in Scholz (1977)]. The monitor also contains facilities for evaluating student responses and managing the response-contingent

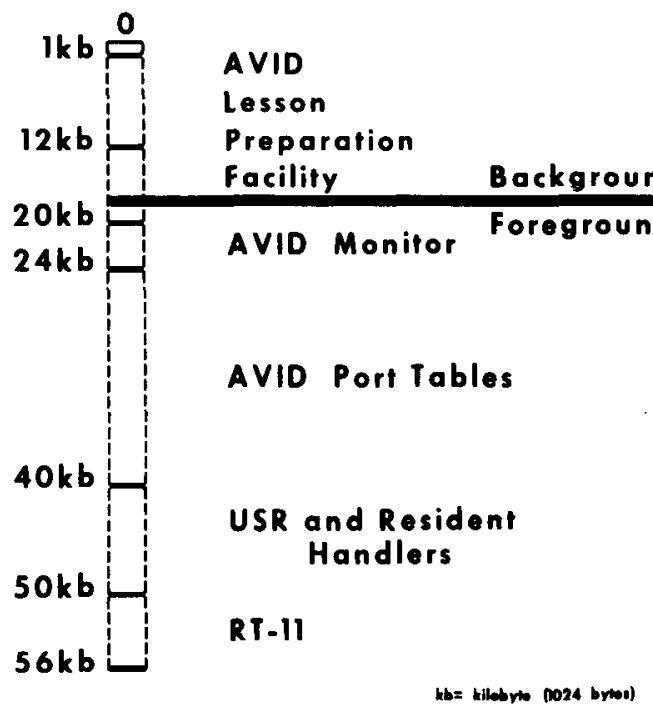

Figure 2. Memory allocation for AVID in a 56K-byte system. 


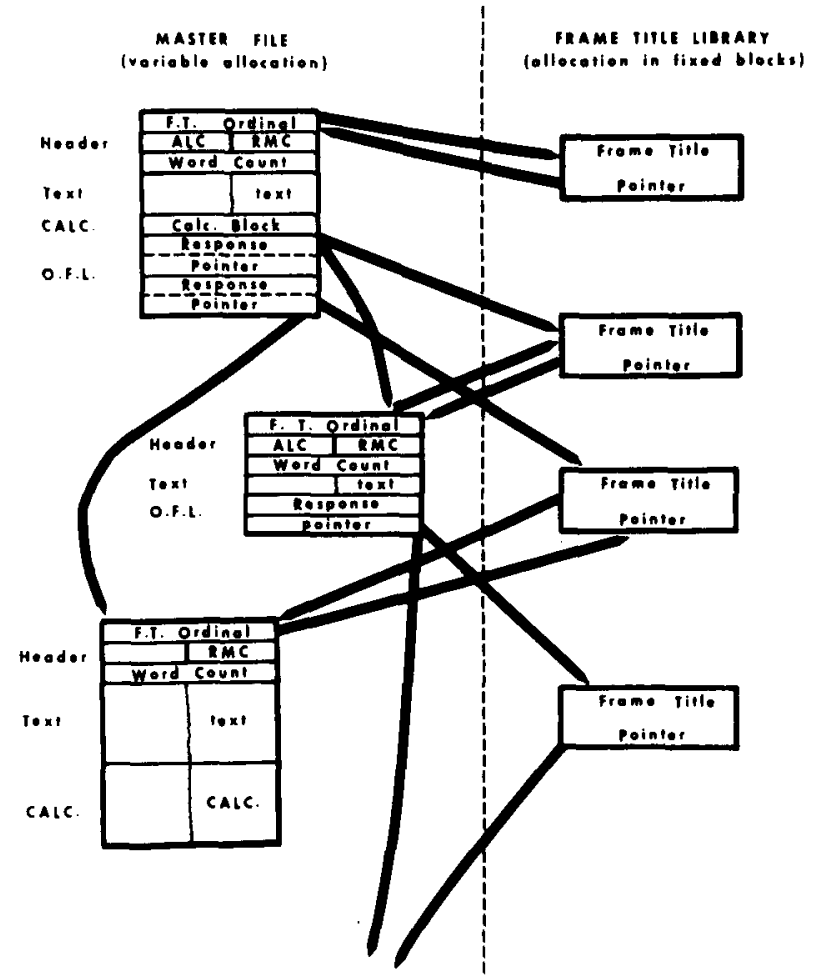

Figure 3. Schematic representation of the data structure for several frames.

transfers from frame to frame. Whenever the monitor recognizes either a command or a question entered by a student, a nonresident overlay is loaded into that student's port table. Once loaded, the overlay executes as a co-routine to the monitor and terminates by passing control either to another overlay or back into the monitor.

\section{Natural Language Processing}

Question answering is processed in two distinct phases. The first phase parses the student's sentence into an ordered collection of keywords which serve as a "probe" into the frame title library. The second phase uses the probe to search FTL in an effort to locate the desired information.

The English parser uses a Markovian grammar which is represented as a path table (Hays, 1967). The table is based on an English vocabulary of around 100 common adjectives, articles, and verbs, and contains a list of legitimate transitions among them. The parsing algorithm is driven by the path table using a simplification of the transition network parser described by Woods (1973). The parser produces an ordered list of keywords (nouns or adjectives) similar in structure to AVID's frame titles.

The ordered keyword list, or "probe," is processed by the second phase, which performs the FTL search. The probe is compared keyword by keyword with each frame title and a "match score," weighted by the length of the probe, is calculated. Those scores that exceed a criterion are retained as a list. When the FTL search is completed, the list is sorted by match score, and the frame with the highest score is displayed as the "answer" to the student's query. If the top several match scores are nearly identical, the associated frame titles are listed to the student as a menu for his selection.

Despite the relative simplicity of the language processing algorithm, its performance as an information retrieval facility is extremely effective. Previous research in computational linguistics has illustrated the degree to which reduction in the scope of the semantic domain leads to simplification in the linguistic analysis (Winograd, 1972). In AVID, the domain is explicitly limited to queries regarding the existence of instructional material within the curriculum. As such, the simple parsing/searching algorithm described above in conjunction with a carefully designed collection of frame titles provides a highly effective retrieval facility.

\section{Student Record Management}

Student access to AVID is controlled by a password file. This file contains each student's password, a set of permission bits, the time of day of the most recent $\log$ on, and the cumulative use time to date. In addition, a separate student record file is associated with each password. If a lesson author desires, he may reserve file space for student record use by any lesson. AVID then reserves the same relative position in each individual student record for use by that lesson. The author then includes a SAVE statement in the calc block of any frame in that lesson, and AVID appropriately maintains the information in the file of any student accessing the lesson. Utility routines can then produce listings of information on all lessons entered by a given student or of all students who have entered a given lesson.

\section{AVID Lesson Preparation Facility}

The Lesson Preparation Facility (LPF) executes as an RT-11 background program. It is entered from any student port (via an "ESC-A" command) and can be used by only one port at a time. Its access is controlled by a dependency queue in the AVID monitor which governs its sequential allocation to various authors on a first-come/first-serve basis. During periods of nonuse, the LPF remains in memory idling until next needed.

Whenever the LPF is entered, it first scans the frame title library in order to produce a listing to the author of any frames that have been named but not completed. It then sequentially prompts the author for a frame title, a text block, a calc block, and a response block. As the information is entered 
by the author, space is allocated in the master file by using an "available storage pointer" which is maintained in the first block. All expressions entered by the author in the calc block are converted from the parenthesized infix notation to a reverse polish intermediate code using conventional compilation algorithms. The intermediate code is interpreted at execution by the AVID monitor. Listings for lesson debugging are produced by disassembling the intermediate code back to source format, thus eliminating the need to waste file space by storing the source code.

One of the AVID monitor overlays is a text editor. This overlay is a simple line-oriented context editor designed to be used for lesson preparation. A source file created with the editor may consist of an entire lesson, a portion of a lesson, or corrections to an existing lesson. Source files created with the editor are submitted to the LPF for compilation. The LPF communicates the result of the compilation back to the author by creating a $\log$ file which indicates any errors detected during compilation.

The editor and the ability of the LPF to process source files greatly simplify the process of lesson transportability. Although there have been several attempts to standardize lesson source listings in order to permit lesson interchange, there exist no universally agreed-upon formats (according to our knowledge). The flexibility of the AVID editor makes it possible to modify CAI lesson source files obtained from other installations into a form acceptable to the AVID LPF. Similarly, an AVID source listing is easily read and understood, thus simplifying its adaptation to other systems.

\section{MOTIVATION FOR THE DESIGN}

The decision to initiate the development of a CAI system was motivated by both pragmatic and academic considerations. The pragmatic motivation grew from the keen recognition of the need for quality CAI in a medical environment. This has been well articulated by other authors (Alpert \& Bitzer, 1970; Brigham \& Kamp, 1973; Hagamen, Linden, Leppo, Bell, \& Weber, 1973; Pengov, Note 1). The systems described and praised by these authors, however, require multimillion-dollar hardware such as the Control Data Cyber 70 or IBM Series 370 computer systems, which are far too expensive for small institutions. Previous attempts to develop relatively inexpensive quality CAI systems have met with marginal acceptance (e.g., DEC's DECAL system). Thus, the stage was set for the design and implementation of a sophisticated system for a small machine.

The pedagogical model on which AVID is based grew from our prior involvement with the educa- tional process. Snow (1972) explored the concept of psychotherapy as an educational process requiring the systematic restructuring of belief systems. The investigation of semantic information structures (Quillian, 1968; Rumelhart, Lindsay, \& Norman, 1972) prompted Scholz to investigate various approaches to the representation of information in memory (Potts \& Scholz, 1975; Scholz, 1972; Scholz $\&$ Potts, 1974). The interdigitation of our orientations led to a view of the educational process as one of systematically incorporating didactic material into previously established information structures. New information is not simply "written" into a passive memory store, but is actively associated or understood in the context of previously existing knowledge. While understanding may be mediated by spatial, affective, or temporal, as well as semantic, associations, we feel that the single most important constituent is the existence of the previous structure.

This view of education places strong emphasis on the need to present information to the student in such a way as to facilitate its incorporation into his weltanschaung. No single author or educator can possibly anticipate the optimal presentation sequence for every student. Rather, the student must be free to seek the information he needs, motivated by his current understanding. Thus, a student can best profit from computerized education if he is free to control the interaction rather than forced to conform to the author's intuitions concerning the organization of information.

Although this view of the learning process is neither totally profound nor totally original, to our knowledge AVID represents the first attempt to incorporate such a view into the design of a CAI system. The efficacy of our approach is an empirical question, and the empirical investigation of the importance of learner control will motivate our first systematic evaluation of student performance.

\section{SUMMARY}

AVID, a general-purpose CAI system implemented on the PDP-11, has been described from three perspectives. From the student's perspective, interaction with AVID can be directed either by student questions or by the sequential flow designed by the lesson author. From an author's perspective, a lesson can be created quickly and efficiently by using AVID's interactive lesson preparation facility. Alternatively, an experienced author has a library of specialized functions at his disposal for use in creating graphics illustrations, maintaining student records, or performing arithmetic calculations. From a system designer's perspective, AVID's data base is developed in a sequentially allocated master file. This file is keyed by the frame title library, a second 
file which associates each frame's title with its origin in the master file. AVID is a timesharing system which permits simultaneous independent interaction with up to eight students (on a $28 \mathrm{~K}$ system).

Once documentation of the system is complete, we plan to formally evaluate a number of its features. Initially, our major interest is in the efficacy of AVID's question-processing facility as a viable educational adjunct.

\section{REFERENCE NOTE}

1. Pengov, R. E. The computer as an aid in medical education. In Proceedings of the Educom Fall Conference (October 16-18, 1974), 1975.

\section{REFERENCES}

AlPERT, D., \& BITZER, D. L. Advances in computer based education. Science, 1970, 167, 1582-1590.

Brigham, C. R., \& Kamp, M. A selected bibliography to computerized instruction in the health sciences. Computers in Biology and Medicine, 1973, 3, 337-342.

Hagamen, W. D., Linden, D., Leppo, M., Bell, W., \& Weber,
J. C. ATS in exposition. Computers in Biology and Medicine, $1973,3,205-226$.

Hays, D. G. Introduction to computational linguistics. New York: American Elsevier, 1967.

Potrs, G. R., \& Scholz, K. W. The internal representation of a three-term series problem. Joumal of Verbal Learning and Verbal Behavior, 1975, 14, 439-452.

Quillian, M. R. Semantic memory. In M. L. Minsky (Ed.), Semantic information processing. Cambridge: MIT Press, 1968

Rumelhart, D. E., Lindsay, P. H., \& Norman, D. A. A process model for long-term memory. In E. Tulving \& W. Donaldson (Eds.), Organization of memory. New York: Academic Press, 1972.

Scholz, K. W. A cognitive approach to the storage and retrieval of structured verbal material. Unpublished doctoral dissertation. Indiana University, 1972.

Scholz, K. W. Timesharing on the PDP-11 under RT-11. Behavior Research Methods \& Instrumentation, 1977, 9, 173-175.

Scholz, K. W., \& Potts, G. R. Cognitive processing of linear orderings. Journal of Experimental Psychology, 1974, 102, 323-326.

Snow, L. H. Contemporary psychiatry. Chicago: Year Book Medical Publishers, 1972.

WinOgRAD, T. Understanding natural language. Cognitive Psychology, 1972, 3, 1-191.

Woods, W. A. An experimental parsing system for transition network grammars. In $\mathrm{R}$. Rustin (Ed.), Natural language processing. New York: Algorithmics Press, 1973. 